\title{
Static charging of graphene and graphite slabs
}

M. Topsakal, and S. Ciraci

Citation: Appl. Phys. Lett. 98, 131908 (2011);

View online: https://doi.org/10.1063/1.3573806

View Table of Contents: http://aip.scitation.org/toc/apl/98/13

Published by the American Institute of Physics

\section{Articles you may be interested in}

Rungs 1 to 4 of DFT Jacob's ladder: Extensive test on the lattice constant, bulk modulus, and cohesive energy of solids

The Journal of Chemical Physics 144, 204120 (2016); 10.1063/1.4948636

Grand canonical electronic density-functional theory: Algorithms and applications to electrochemistry

The Journal of Chemical Physics 146, 114104 (2017); 10.1063/1.4978411

Large work function reduction by adsorption of a molecule with a negative electron affinity: Pyridine on ZnO(10) 10$)$

The Journal of Chemical Physics 139, 174701 (2013); 10.1063/1.4827017

The response of mechanical and electronic properties of graphane to the elastic strain Applied Physics Letters 96, 091912 (2010); 10.1063/1.3353968

A climbing image nudged elastic band method for finding saddle points and minimum energy paths The Journal of Chemical Physics 113, 9901 (2000); 10.1063/1.1329672

On the large-gradient behavior of the density functional exchange energy

The Journal of Chemical Physics 85, 7184 (1998); 10.1063/1.451353

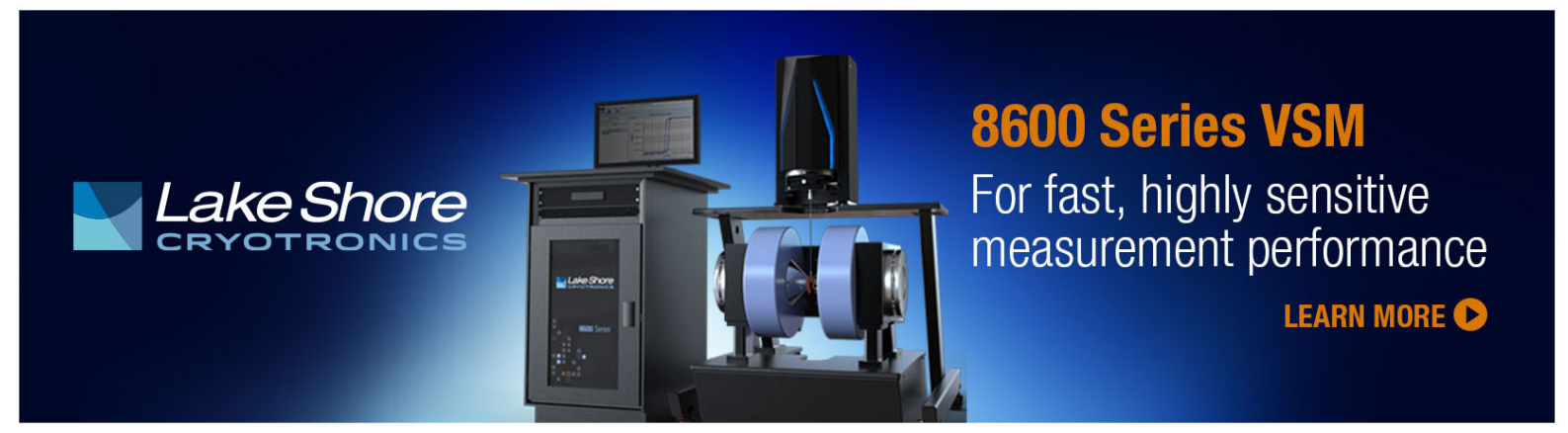




\title{
Static charging of graphene and graphite slabs
}

\author{
M. Topsakal ${ }^{1}$ and S. Ciraci ${ }^{1,2, a)}$ \\ ${ }^{1}$ UNAM-Institute of Materials Science and Nanotechnology, Bilkent University, Ankara 06800, Turkey \\ ${ }^{2}$ Department of Physics, Bilkent University, Ankara 06800, Turkey
}

(Received 31 July 2010; accepted 14 March 2011; published online 31 March 2011)

\begin{abstract}
The effect of external static charging of graphene and its flakes are investigated by using first-principles calculations. While the Fermi level of negatively charged graphene rises and then is quickly pinned by the parabolic, nearly free electronlike bands, it moves down readily by removal of electrons from graphene. Excess charges accumulate mainly at both surfaces of graphite slab. Even more remarkable is that Coulomb repulsion exfoliates the graphene layers from both surfaces of positively charged graphite slab. The energy level structure, binding energy, and spin-polarization of specific adatoms adsorbed to a graphene flake can be monitored by charging. (C) 2011 American Institute of Physics. [doi:10.1063/1.3573806]
\end{abstract}

Graphene ${ }^{1}$ is a semimetal having conduction and valance bands which cross linearly at the Fermi level $\left(E_{F}\right)$. The resulting electron-hole symmetry reveals itself in an ambipolar electric field effect, whereby under bias voltage the charge carriers can be tuned continuously between electrons and holes in significant concentrations. Excess electrons and holes can also be achieved through doping with foreign atoms. ${ }^{2-4}$ For example, adsorbed alkali atoms tend to donate their valence electrons to $\pi^{*}$-bands of graphene. The excess electrons results in the metalization of graphene. ${ }^{5}$ Hole doping is achieved by the adsorption of bismuth or antimony. ${ }^{6}$ However, the system remains electrically neutral through either way of doping. Recently, carrier concentration and spatial distribution of charge are also changed for very short time intervals by photoexcitation of electrons from the filled states leading to the photoexfoliation of graphite. ${ }^{7-9}$

In this letter, we demonstrate that the properties of graphene can be modified either by direct electron injection into it or electron removal from it; namely, by charging the system externally. Remarkably, the Coulomb repulsion exfoliates the graphene layers from both surfaces of positively charged graphene slab. This result may be exploited to develop a method for intact exfoliation of graphene. In addition to exfoliation, the energy level structure, density of states, ${ }^{10}$ binding energies, and desorption of specific adatoms can be monitored by charging.

Our results are predicted through first-principles plane wave calculations carried out within density functional theory (DFT) using projector-augmented wave potentials. ${ }^{11}$ The exchange correlation potential is approximated by localdensity approximation (LDA). We also performed GGA $+\mathrm{vdW}$ (generalized gradient approximation including van der Waals corrections ${ }^{12}$ ) for a better account of VdW interlayer interactions between graphite slabs. A plane-wave basis set with kinetic energy cutoff of $500 \mathrm{eV}$ is used. All atomic positions and lattice constants are optimized by using the conjugate gradient method, where the total energy and atomic forces are minimized. The convergence for energy is chosen as $10^{-5} \mathrm{eV}$ between two steps, and the maximum force allowed on each atom is less than $0.01 \mathrm{eV} / \AA$. The Brillouin zone is sampled by $(15 \times 15 \times 5)$ special k-points

${ }^{a)}$ Electronic mail: ciraci@fen.bilkent.edu.tr. for primitive unit cell. Calculations for neutral, as well as charged systems are carried out by using VASP package. ${ }^{13}$ Two-dimensional graphene is treated within periodic boundary conditions using the supercell method having more than $50 \AA$ separation between adjacent layers. The amount of charging, $\rho$, is specified as either positive charging, i.e., electron depletion $(\rho>0)$ or negative charging, i.e., excess electrons, in units of \pm electron (e) per carbon atom or per unit cell. For charged calculations, additional neutralizing background charge is applied. ${ }^{14}$

The work function of neutral graphene is calculated to be $4.77 \mathrm{eV}$. Lowest two parabolic bands $\Psi_{5}$ and $\Psi_{6}$ in Fig. 1(b) have effective masses $\mathrm{m}^{*}=1.05$ and $1.02 \mathrm{~m}_{e}$ (free electron mass) in the $x y$-plane parallel to the atomic plane of graphene. Hence they are nearly free electronlike (NFE) in two dimensions, but they are bound above the graphene plane. As shown, in Figs. 1(d) and 1(f), these "surface" states $^{15}$ can be expressed as $\Psi_{S} \sim e^{i \mathbf{k}_{\|}} \cdot \mathbf{r}_{\|} \Phi(z)$, where $\mathbf{r}_{\|}$and $\mathbf{k}_{\|}$ are in the $x y$-plane. Parabolic bands at higher energies become NFE in three dimensions. When the electrons are removed, the Fermi level is lowered from the Dirac point and positively charged graphene attains metallic behavior as in Fig. 1(a). At the end, the work function increases. However, under negative charging, whereby electrons are injected to the graphene, Fermi level raises above the Dirac point and eventually becomes pinned by NFE parabolic bands as in Fig. 1(c). These parabolic NFE bands start to get occupied around $\rho=-0.0115 \mathrm{e} /$ atom (or surface excess charge density $\sigma=-0.0710 \mathrm{C} / \mathrm{m}^{2}$ ). Upon charging the bound charge of $\Psi_{S}$ states is further removed from graphene as shown in Fig. 1(f). This situation can be interpreted as the excess electrons start to spill out toward vacuum. Figure $1(\mathrm{~g})$ shows another important effect of charging where the lattice constants increase with positive charging. On the other hand, negative charging has little effect on lattice constants, since the excess electrons mostly spill out.

The effect of charging on a graphite slab consisting of three layers of graphene is better seen in Fig. 2. When negatively charged, the excess electrons are mainly accumulated on both surfaces, but with smaller amount at the middle layer. The effect of charging on structure is minute, since the bonds are intact and the excess electrons rapidly spill out toward the vacuum. However, the situation is dramatically 
(a) $\rho=+0.20$ (e/atom)

(b) neutral

(c) $\rho=-0.05$ (e/atom)
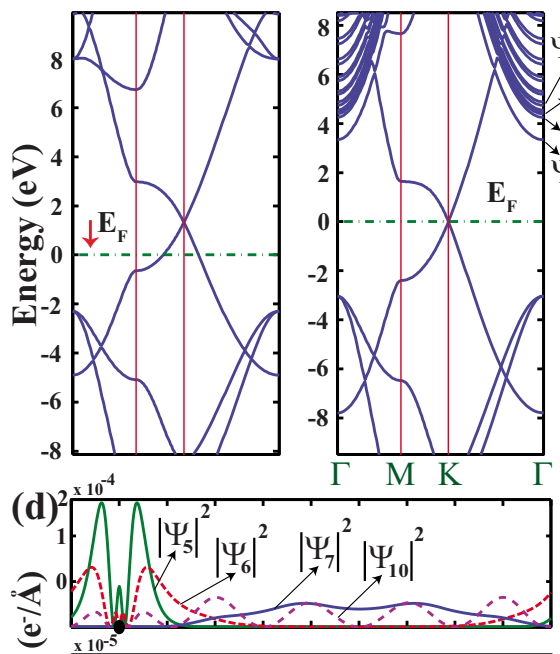

(e)
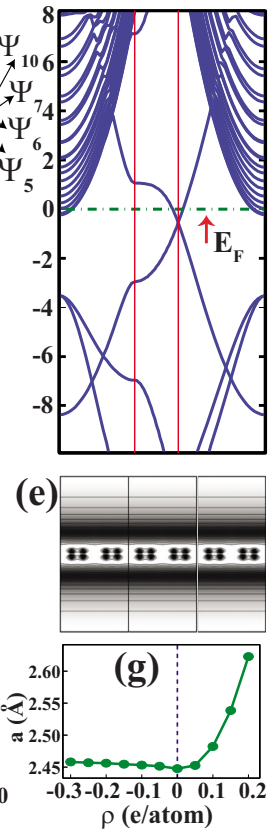

(f)

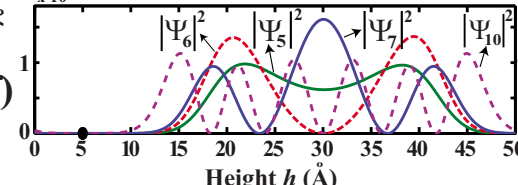

FIG. 1. (Color online) Energy band structure of charged and neutral graphenes. (a) Positively charged graphene by $\rho=+0.20$ e/atom. (b) Neutral. (c) Negatively charged graphene by $\rho=-0.05 \mathrm{e} /$ atom, where excess electrons start to occupy the surface states. Zero of energy is set to Fermi level. (d) Planarly averaged charge density $(\lambda)$ of states, $\Psi_{5-10}$, of neutral graphene. (e) Charge contour plots of the lowest surface state, $\Psi_{5}=\Psi_{S}$ in a plane perpendicular to graphene. (f) Same as (d) after charging with $\rho=-0.05 \mathrm{e} /$ atom. (g) Variation in lattice constant $a$ of graphene as a function of charging.

different for the case of positive charging. The charge isosurfaces in Fig. 2 show that positive charge, occurs mainly on both surfaces (i.e., first and third graphene layers), whereas the middle graphene has relatively small positive charge. This is an expected result for a metallic system. The interlayer interaction in the neutral three-layer slab is attractive

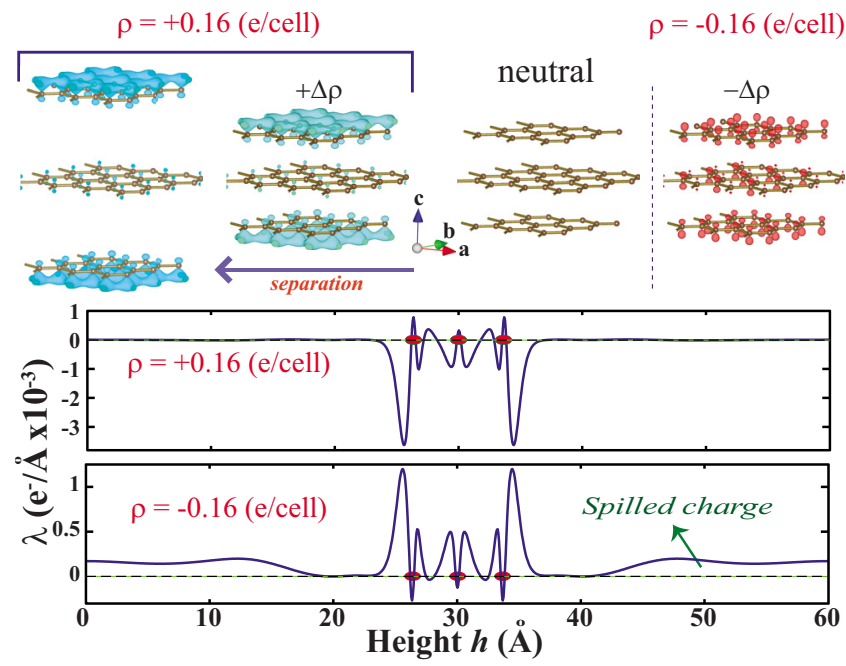

FIG. 2. (Color online) Exfoliation of graphene layers from both surfaces of a three-layer graphite slab (in AB-stacking) caused by electron removal. Isosurfaces of difference charge density, $\Delta \rho$, show the electron depletion. The excess charge on the negatively charged slab is not sufficient for exfoliation. The distributions of planar averaged charge density $(\lambda)$ perpendicular to the graphene plane are shown below both for positive and negative charging (calculations are performed by GGA+vdW). and is calculated to be 17 (36) $\mathrm{meV} /$ atom calculated by LDA $(\mathrm{GGA}+\mathrm{vdW})$, which becomes even weaker upon depopulation of $\pi$-orbitals. GGA $+\mathrm{vdW}$ calculations predict that a threshold charge, $\mathrm{Q}=0.16 \mathrm{e} /$ cell gives rise to exfoliation of two outermost layers. LDA calculations yield relatively lower threshold charge of $\mathrm{Q}=0.14$ e/cell. We also performed a systematic analysis of exfoliation for thicker slabs consisting of five to ten layers of graphene. We found that the threshold charge increases with increasing slab thickness. However, our analysis based on the planar averaged charge densities suggests that the exfoliation of outermost layers occurs when approximately the same amount of positive charge is accumulated on the outermost layers. For example, the exfoliation of three-layer and six-layer graphene flakes take place when their outermost layers have positive charge of $0.065 \mathrm{e} / \mathrm{cell}$ and $0.066 \mathrm{e} / \mathrm{cell}$, respectively. On the other hand, increasing of threshold charge by going from threelayer to six-layer occurs due to the charge spill to the inner layers. This situation can be explained by a simple electrostatic model, where the outermost layers of slabs is modeled by uniformly charged planes, which yield repulsive interaction independent of their separation distance, i.e., $F$ $\propto q^{2} /\left(A \cdot \epsilon_{0}\right)$, where $q$ is excess positive charge per unit cell with the area $A$. Nonetheless, these values of charging are quite high and can be attained in small flakes locally by the tip of scanning tunneling microscope.

Ultrafast graphene ablation was directly observed by means of electron crystallography. ${ }^{7}$ Carriers excited by ultrashort laser pulse transfer energy to strongly coupled optical phonons. Graphite undergoes a contraction, which is subsequently followed by an expansion leading eventually to laser-driven ablation. ${ }^{7}$ Much recently, the understanding of photoexfoliation have been proposed, where exposure to femtosecond laser pulses has led to athermal exfoliation of intact graphenes. ${ }^{8}$ Based on time dependent DFT (TD-DFT) calculations, it is proposed that the femtosecond laser pulse rapidly generates hot electron gas at $\sim 20.000 \mathrm{~K}$, while graphene layers are vibrationally cold. The hot electrons are spill out, leaving behind a positively charged graphite slab. The charge deficiency accumulated at the top and bottom surfaces leads to athermal excitation. ${ }^{8}$ The exfoliation in static charging described in Fig. 2 is in compliance with the understanding of photoexcitation revealed from TD-DFT calculations, since the driving force which leads to the separation of graphenes from graphite is related mainly with electrostatic effects in both process.

The effects of charging become emphasized when the size of graphene flake is small. In this respect, the flake behaves like a quantum dot and hence energy level structure is affected strongly. The flake we consider has a rectangular shape and hence it consists of armchair, as well as zigzag edges as shown in Fig. 3. It is therefore antiferromagnetic ground state when neutral. Isosurfaces of difference charge density, $\Delta \rho$, of the same flake for three different charge states are shown in Fig. 3(a). $\Delta \rho$ is calculated by subtracting the total charge density of the neutral flake from that of charged ones. For a better comparison, charge density of the neutral flake is calculated using the atomic structure of the charged ones. It is seen that the edge states due to zigzag edges are most affected from charging. In Fig. 3(a), while charge is depleted mainly from edge states, excess electrons are accumulated predominantly at the zigzag edges. As shown in Fig. 


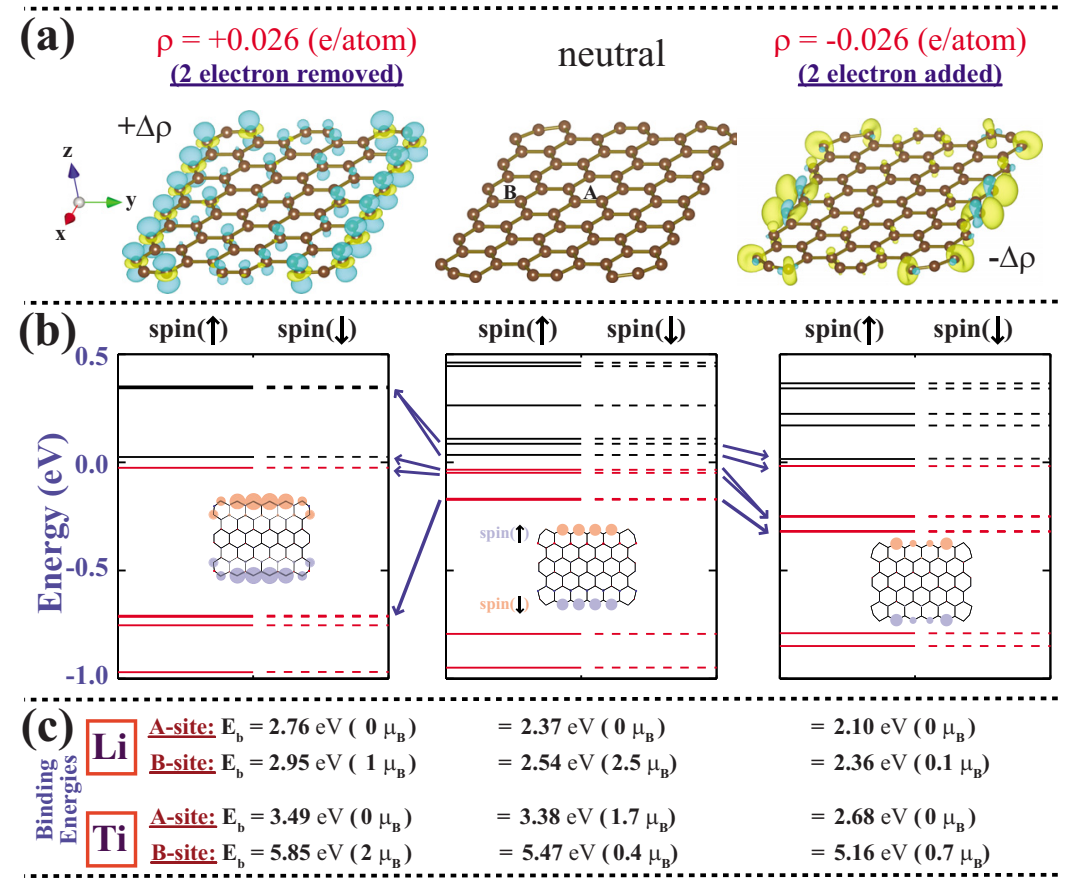

FIG. 3. (Color online) Effect of charging on graphene flake consisting of 78 carbon atoms. (a) Isosurfaces of difference charge density $\Delta \rho$ of positively charged, neutral, and negatively charged slabs. (b) Corresponding spin-polarized energy level structure. Solid and continuous levels show spin up and spin down states. Distribution of magnetic moments at the zigzag edges are shown by insets. Zero of energy is set to Fermi level. (c) Variation in binding energy and net magnetic moment of specific adatoms adsorbed in two different positions, namely, A-site and B-site indicated in (a). 3(b), while the antiferromagnetic state of the flake is unaltered, charging causes emptying and filling of highest occupied molecular orbital and lowest unoccupied molecular orbital states, changing of level spacings and their energies relative to vacuum level. Additionally, magnetic moments of zigzag edge atoms are strongly affected depending on the sign of charging in Fig. 3(b). In particular, the binding energies and magnetic moments of specific adatoms depend on its position and charging of the flake. In Fig. 3(c) we consider $\mathrm{Li}$ and $\mathrm{Ti}$, which normally adsorbed to graphene by donating charge. Generally, the binding energies increases (decreases) with positive (negative) charging. We also found that the effects of monopole and dipole corrections on the effects of charging on the binding energies is minute. For example, the binding energy of $\mathrm{Li}$, when two electrons are removed, increases from 2.756 to 2.764 upon corrections. However, the effect of charging becomes more pronounced when the adatom is placed close to the edge of positively charged flake since the additional charges are mostly confined at the edges. Similarly, the magnetic moment at the adatom site varies depending on the adsorption site and charging state of the flake.

In summary, we revealed the dramatic effects of static and external charging of graphene and its flake. Charging through electron depletion of graphite surfaces leads to exfoliation of graphene. We also show that the binding energy and local magnetic moments of specific adatoms can be tuned by charging.
We thank the DEISA Consortium (www.deisa.eu), funded through the EU FP7 Project No. RI-222919, for support within the DEISA Extreme Computing Initiative. We acknowledge partial financial support from The Academy of Science of Turkey (TUBA).

${ }^{1}$ K. S. Novoselov, A. K. Geim, S. V. Morozov, D. Jiang, Y. Zhang, S. V. Dubonos, I. V. Grigorieva, and A. A. Firsov, Science 306, 666 (2004).

${ }^{2}$ X. Wang, X. Li, L. Zhang, Y. Yoon, P. K. Weber, H. Wang, J. Guo, and H. Dai, Science 324, 768 (2009).

${ }^{3}$ T. O. Wehling, K. S. Novoselov, S. V. Morozov, E. E. Vdovin, M. I. Katsnelson, A. K. Geim, and A. I. Lichtenstein, Nano Lett. 8, 173 (2008).

${ }^{4}$ H. Sevinçli, M. Topsakal, E. Durgun, and S. Ciraci, Phys. Rev. B 77, 195434 (2008).

${ }^{5}$ C. Ataca, E. Aktürk, S. Ciraci, and H. Ustunel, Appl. Phys. Lett. 93, 043123 (2008).

${ }^{6}$ I. Gierz, C. Riedl, U. Starke, C. R. Ast, and K. Kern, Nano Lett. 8, 4603 (2008).

${ }^{7}$ F. Carbone, P. Baum, P. Rudolf, and A. H. Zewail, Phys. Rev. Lett. 100, 035501 (2008).

${ }^{8}$ Y. Miyamoto, H. Zhang, and D. Tomanek, Phys. Rev. Lett. 104, 208302 (2010).

${ }^{9}$ R. K. Raman, Y. Murooka, C.-Y. Ruan, T. Yang, S. Berber, and D. Tomanek, Phys. Rev. Lett. 101, 077401 (2008).

${ }^{10}$ K. T. Chan, H. Lee, and M. L. Cohen, Phys. Rev. B 83, 035405 (2011).

${ }^{11}$ P. E. Blöchl, Phys. Rev. B 50, 17953 (1994).

${ }^{12}$ S. Grimme, J. Comput. Chem. 27, 1787 (2006).

${ }^{13}$ G. Kresse and J. Furthmuller, Phys. Rev. B 54, 11169 (1996).

${ }^{14}$ G. Makov and M. C. Payne, Phys. Rev. B 51, 4014 (1995).

${ }^{15}$ M. Posternak, A. Baldereschi, A. J. Freeman, and E. Wimmer, Phys. Rev. Lett. 52, 863 (1984). 